Proceedings

\title{
Pattern-Based Approaches to Evaluating Water Quality $^{\dagger}$
}

\section{L. Marrin}

Water Sciences \& Insights, POB 232125, Encinitas, CA 92023, USA; marrin@watersciences.org

+ Presented at the 2nd International Electronic Conference on Water Sciences, 16-30 November 2017; Available online: http://sciforum.net/conference/ecws-2.

Published: 16 November 2017

\begin{abstract}
Beyond just a visual data enhancement of water quality data, the use of hierarchically arranged spatial patterns and sequentially arranged temporal patterns can provide a means of discerning anomalies and correlations among water quality parameters that would otherwise be difficult to distinguish or communicate. Similarly, comparing actual water quality patterns with ideal patterns (generated by models) or abstract patterns (created from hypotheses) can be used to evaluate their relevance to observed datasets. Finally, assessing the connectivity of water quality elements as a coherent network, rather than as isolated data points, can expose feedback loops, disproportionate effects and data limitations.
\end{abstract}

Keywords: water; quality; patterns; spatial; temporal; communication

\section{Introduction}

As water quality evaluations continue to increase their number of analytes and frequency of measurements, a challenge arises as how to present these data in a manner that can best be used to implement remedial actions and inform water resource decisions. Graphic artists have developed visualization techniques using colors, textures, and layers that enhance people's accessibility to and understanding of data. However, beyond just visual data enhancement, the use of hierarchically arranged spatial patterns and sequentially arranged temporal patterns can provide an accessible means of discerning trends, anomalies, and correlations among water quality parameters that otherwise may be difficult to distinguish or to communicate.

Researchers have found that people recognize patterns more easily than they do words, numbers or symbols, apparently because patterns are fundamental to the natural world and have served (evolutionarily) as indicators of environmental threats or resources. In fact, the processing of patterns is considered by some neuroscientists to be the essence of an evolved human brain, which is able to recognize and project abstract patterns that underlie language, music, art, and science [1]. This is reportedly the case for both spatial patterns (e.g., shapes, geometries, relative positions) and temporal patterns (e.g., rhythms, vibrations, cycles), some of which elude people's conscious awareness or understanding. For example, fractals possessing a dimension, or tortuosity, similar to those appearing in the natural world (e.g., clouds, waves, trees) are preferred by people whether or not they have any concept of fractals or can even identify one [2]. A fractal is a pattern that repeats over a range of scales, as is shown on Figure 1. Similar to fractals, people can recognize topological depictions of network patterns even when they are stretched, twisted, or otherwise altered. 


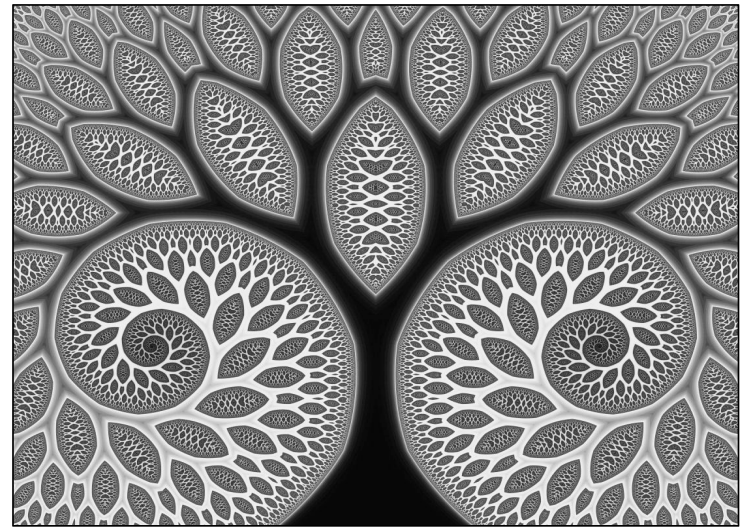

(a)

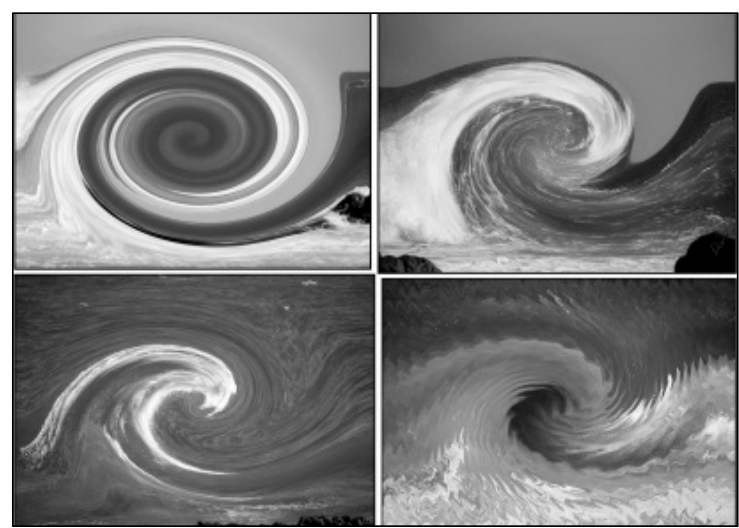

(b)

Figure 1. Examples of a fractal pattern (a) and an altered wave pattern (b) from 123RF.

Do humans impose patterns on the natural world or is nature replete with patterns? Because our brains essentially construct facsimiles of the world via our senses, memory, and other inputs, this question has long been contemplated. Apparently, myriad dissimilar processes (e.g., biological chemical, geological, hydrological) generate the remarkably similar patterns that we perceive in nature. It has been hypothesized that there is no scientific law of pattern formation, but rather a common recipe for pattern creation that takes into account non-equilibrium conditions, nonlocal events and thresholds for change [3]. Explaining spatial or temporal patterns through the analysis of their components and processes (reductively) is difficult because patterns frequently emerge as a property of entire systems. As such, systems theory represents a viable approach to water quality, although its requisite networks are among the most abstract renditions of patterns.

\section{Pattern-Based Approaches}

Conventional approaches to investigating water quality employ simplifying assumptions for the relevant variables and processes in order to display datasets or to model complex aquatic systems. Principal component analysis (PCA) has served as a valuable technique for exploring correlations among water quality indicators in rivers and lakes, as well as answering questions about spatiotemporal patterns and the diverse sampling regimes that generate them [4]. Similarly, advective-diffusive mathematical models designed to simulate temporal changes in water quality parameters have yielded three-dimensional spatial predictions by focusing on a chosen subset of parameters and processes occurring in lakes and reservoirs [5].

Systems theory describes the interactions among water system elements as spatiotemporal relationships, or patterns. Whereas matter and energy are cycled through a system, the focus is on describing a system's organization and interactions, thus permitting the identification of controls, hierarchies, and feedback dynamics that govern its trajectory and observable behavior. Evaluating the connectivity of water quality parameters as a coherent network, rather than as isolated data points, has been used to expose data limitations and changing relationships among the system's elements that influence water quality. Systems approaches have also been applied to setting water quality standards, selecting variables to assess nonlinearity and spatiotemporal variability, and optimizing community-based programs designed to monitor water quality [6,7].

Finally, simple pattern-based approaches have been used to compare actual water quality patterns with ideal patterns (e.g., generated by models) and abstract patterns (e.g., created from theories) to assess their practicality and relevance to observed datasets. This approach is useful for communicating or interpreting water quality data for non-scientists (e.g., stakeholders, managers), who often misinterpret or are confused by the mathematics, symbols, and graphics appearing in technical reports and journal articles [8]. Schematics for some of the pattern-based relationships that will be discussed in the next section are shown on Figure 2. 


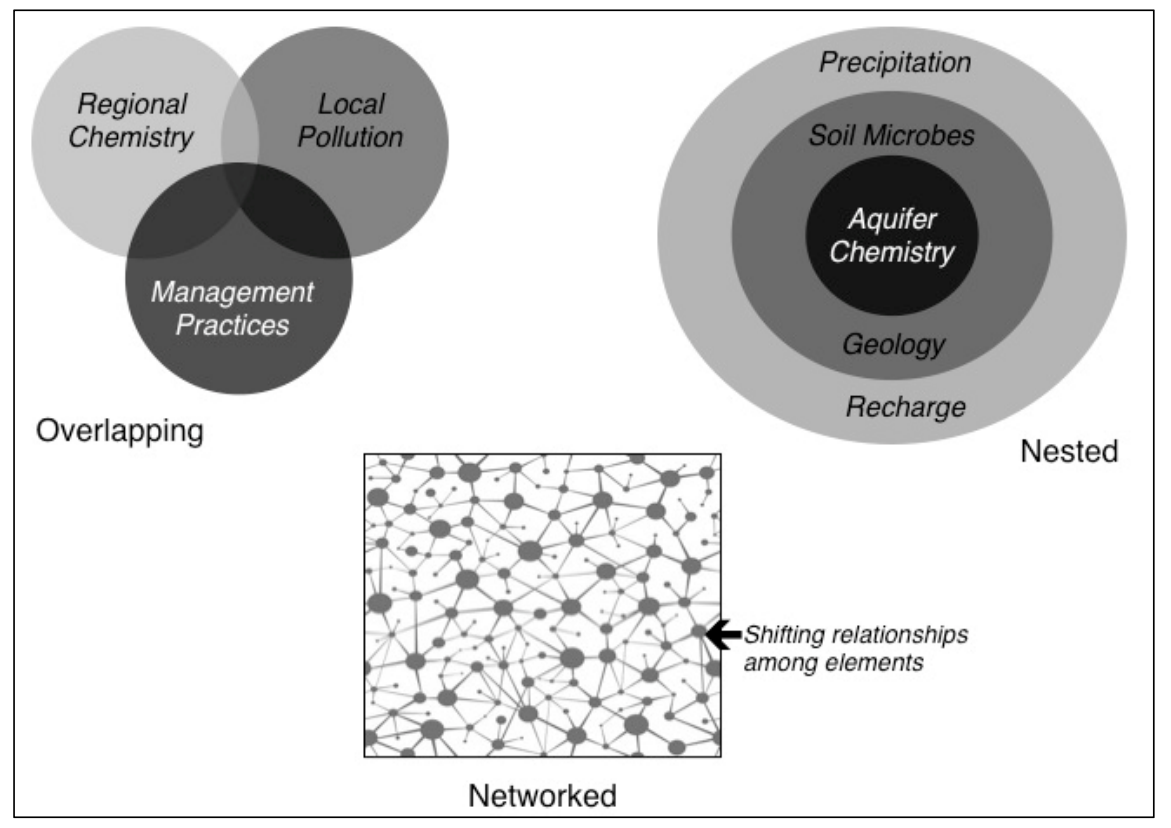

Figure 2. Schematic representations for three pattern relationships described in this presentation.

\section{Discussion}

In assessing patterns of water quality as a function of the factors affecting them, some of the most common factors include natural cycles or episodic events and anthropogenic influences. The impact of rivers on groundwater quality is an example of the first factor and was recently described for an alluvial plain, whereby the recharge from rivers within the watershed resulted in both direct and indirect effects [9]. Spatial patterns of nitrate concentrations in groundwater were related to their dilution by the basin-wide infiltration of river water (directly) and by the river's effects on alluvial soils that supported the denitrification of recharged water (indirectly) in specific regions. Essentially, the spatiotemporal patterns of river recharge were responsible, at least in part, for the spatial patterns of nitrate observed in the underlying aquifer.

The operation of dams is an example of a human designed influence on the water quality of a river. Spatial changes in water quality patterns located downstream from dams are often due to seasonal releases or outflows. Seasonal patterns of outflow from a dam were able to alter nitrogen, phosphorus, and chlorophyll concentrations as far as $60 \mathrm{~km}$ downstream of the dam [10]. Reduced temperatures in the river resulting from the release of cold bottom waters varied as a function of seasonal temperature cycles in the river system. Temporal patterns for reservoir releases were contrasted with the spatial patterns of river water quality as a means of suggesting reservoir management strategies that could minimize impacts to downstream ecosystems.

Patterns of water quality can influence, as well as be influenced by, ecological factors. For example, spatiotemporal maps of regional-scale water quality parameters (e.g., temperature, $\mathrm{pH}$, turbidity, and electrical conductivity) in the southeastern region of the USA displayed a correlation with regional ecosystem divisions and the gross distribution of fishes [11]. Whereas this relationship did not hold for all regions, a comparison of these vastly different patterns provided an indication as to whether relationships were robust enough to compensate for the limited number of parameters considered. While explanations for the correlative relationship (e.g., aquatic ecosystem responses to terrestrial ecosystem characteristics or changes) were not always apparent, comparing the patterns suggested where further investigation was warranted.

Water quality patterns associated with terrestrial development are often scale-variant and related to factors that depend more on geography and topography than on land use itself [12]. For example, urban sprawl and lake water quality in China were investigated on the basis of 27 water quality and terrestrial parameters (e.g., percent urbanization, watershed slope, patch density, major ions, metals, and organic carbon). Overlaying the various patterns indicated that landscape features 
had a greater influence on lake water than did land use. These types of pattern comparisons represent an interesting means of evaluating where managers and regulators should place their emphasis (e.g., land use versus watershed location) in addressing water quality degradation.

As patterns are sometimes arranged hierarchically or within one another, evaluating water quality issues by assessing patterns on progressively larger or smaller scales or as nested cycles (temporally) and distributions (spatially) can be informative. Landfill leachate (aqueous) is a source of pollutants to groundwater aquifers and a contributor of polyfluoroalkyl substances (PFASs) [13]. The presence and concentration of PFASs in landfill leachate differed on the basis of flow rates, location, precipitation, and water chemistry dynamics (i.e., suspended solids, dissolved solids, $\mathrm{pH}$, organic carbon). Hence, PFASs loading to the leachate exhibited a spatiotemporal pattern reflecting chemical cycles in the water and soil, as well as the spatial distribution of landfill soils receiving wastes and supporting conditions appropriate for pollutant degradation or sorption.

There are number of human activities (e.g., data selection/collection) and natural phenomena that create conditions capable of influencing how data patterns relate to one another. Table 1 lists examples of relationships that have been discussed in this section. Many of these examples could fit multiple pattern types or relationships, but the most obvious were selected. A next step in assessing patterns may focus on the scientific reasoning (perhaps contrasted in a manner similar to that of data elucidation) that is used to formulate hypotheses and projections for water, thus making them more intelligible to decision-makers and the public. An evaluation of reasoning patterns has been recommended for non-scientists as a way to assess technical assertions [14].

Table 1. Examples of various pattern relationships as related to type (spatial and/or temporal).

\begin{tabular}{cccc}
\hline Pattern Type & Overlapping & Nested & Networked \\
\hline Spatial & Land uses & Watersheds & Sampling points \\
Temporal & Flood events & Nutrient cycles & Evolving graphs \\
Spatiotemporal & Concentration gradients & Hierarchical models & Groundwater withdrawals \\
\hline
\end{tabular}

\section{Conclusions}

Despite the diversity of water quality topics and objectives described in the previous examples, at least one similarity among them is that interactions among pertinent parameters can be displayed as networked, overlapping, hierarchical, nested or other pattern arrangements, thus rendering them more transparent to professionals and the public. Pattern descriptions and details are frequently communicated more understandably via visuals and even auditory means than through abstract symbols and equations. The comparison of patterns alone is seldom adequate for discerning causal relationships involving water quality, requiring investigations into whether and how the patterns are actually related and into the potential mechanisms underlying those relationships. Most of the pattern analysis and comparison methods described in this short presentation are based on a vast array of simplifying assumptions that are required to even posit such mechanisms.

Acknowledgments: Water Sciences \& Insights funded the preparation of this manuscript.

Conflicts of Interest: The author declares no conflict of interest.

\section{References}

1. Mattson, M.P. Superior pattern processing is the essence of the evolved human brain. Front. Neurosci. 2014, $8,1-17$.

2. Taylor, R.P.; Spehar, B. Fractal fluency: An intimate relationship between the brain and processing of fractal stimuli. In The Fractal Geometry of the Brain; Di Ieva, A., Ed.; Springer Nature: New York, NY, USA, 2016; Chapter 30.

3. Ball, P. Patterns in Nature; University of Chicago Press: London, UK, 2016.

4. Sergeant, C.J.; Starkey, E.E.; Bartz, K.K.; Wilson, M.A.; Mueter, F.J. A practitioner's guide for exploring water quality patterns using principal components analysis and procrustes. Environ. Monit. Assess. 2016, 188, doi:10.1007/s10661-016-5253-z. 
5. Hashemi Monfared, S.A.; Mirbagheri, S.A.; Sadrnejad, S.A. A three-dimensional, integrated seasonal separate advection-diffusion model to predict water quality patterns in the Chahnimeh Reservoir. Environ. Model. Assess. 2014, 19, 71-83.

6. Patten, B.C. System theory formulation of site-specific water quality standards and protocols. Ecol. Model. 1984, 23, 313-340.

7. Burgos, A.; Páez, R.; Carmona, E.; Rivas, H. A systems approach to modeling community-based environmental monitoring: A case of participatory water quality monitoring in rural Mexico. Environ. Monit. Assess. 2013, 185, 10297-10316.

8. Marrin, D.L. Enhancing interactions between artists and scientists via a common language. Acad. J. Sci. 2013, 2, 511-522.

9. Baillieux, A.; Campisi, D.; Jammet, N.; Bucher, S.; Hunkeler D. Regional water quality patterns in an alluvial aquifer: Direct and indirect influences of rivers. J. Contam. Hydrol. 2014, 169, 123-131.

10. Westhorpe, D.P.; Mitrovic, S.M.; Growns, I.O.; Hadwen, W.L.; Rees, G.N. Disruption in water quality patterns along the river continuum by a large bottom release dam. Australas. J. Environ. Manag. 2015, 22, 400-416.

11. McDaniel, T.W.; Hunsaker, C.T.; Beauchamp, J.J. Determining regional water quality patterns and their ecological relationships. Environ. Manag. 1987, 11, 507-518.

12. Sun, Y.; Guo, Q.; Liu, J.; Wang, R. Scale effects on spatially varying relationship between urban landscape patterns and water quality. Environ. Manag. 2014, 54, 272-287.

13. Benskin, J.P.; Li, B.; Ikonomou, M.G.; Grace, J.R.; Li, L.Y. Per- and polyfluoroalkyl substances in landfill leachate: patterns, time trends, and sources. Environ. Sci. Technol. 2012, 46, 11532-11540.

14. Stemwedel, J.D. Evaluating scientific claims (or, do we have to take the scientist's word for it?). Scientific American, 30 September 2011. Available online: https://scientificamerican.com (accessed on 5 October 2017). 